\title{
GEOTRACES
}

\section{A GLOBAL STUDY OF THE MARINE BIOGEOCHEMICAL CYCLES OF TRACE ELEMENTS AND THEIR ISOTOPES}

\author{
BY ROBERT F. ANDERSON AND GIDEON M. HENDERSON
}

Since the late 1990s there has been a growing impetus for a comprehensive global study of the marine biogeochemical cycles of trace elements and their isotopes (TEIs). This has led to the development of a research program known as GEOTRACES.

GEOTRACES is motivated by the fact that TEIs play critical roles in many aspects of oceanography, and by the incompleteness of our understanding of their marine biogeochemical cycles. Better knowledge of the distribution and behavior of TEIs in seawater will provide cidate the mechanisms that control the fate of pollutants in the oceans. Some TEIs, particularly radionuclides, constrain rates of key processes regulating the carbon cycle. Other TEIs can be used to study mixing processes in the ocean on time scales not amenable to direct observation. TEI distributions in marine sediments provide many of the proxies used to reconstruct past environmental conditions (e.g., productivity, circulation, ecosystem structure, weathering, hydrothermal activity, anoxia).

Despite these important roles and

\section{Since the late 1990s there has been a growing} impetus for a comprehensive global study of

\section{the marine biogeochemical cycles of trace elements and their isotopes (TEIs).}

insights into a wide range of oceanic processes. It will provide, for example, understanding of the role that micronutrients play in regulating ecosystem structure and productivity and will elu- uses of TEIs, knowledge of their cycles is limited. For example, the distributions, sources, and sinks of micronutrients are so poorly known that their sensitivity to global change and the resulting changes in marine ecosystems and the carbon cycle cannot be meaningfully predicted. Similarly, although it was established decades ago that many TEIs are removed from seawater by sedimentation, scientists still lack quantitative rates for, and a mechanistic understanding of, these processes. It is also clear that sediment TEI distributions relate to climate variability, but our ability to use TEIs as reliable proxies is limited by incomplete characterization of their current biogeochemical cycles. This lack of knowledge limits our ability to test ocean models against past conditions, and therefore limits our ability to forecast changes.

Marine geochemists are poised to make significant progress in TEI biogeochemistry. Advances in clean sampling protocols and analytical techniques

Robert F. Anderson (boba@ldeo.columbia.edu) is Doherty Senior Scholar and Associate Director, Geochemistry, LamontDoherty Earth Observatory of Columbia University, Palisades, NY, USA. Gideon

M. Henderson is University Lecturer in Environmental Earth Sciences, University of Oxford, Oxford, England. 
provide unprecedented capability for measurement of a wide range of TEIs. The potential afforded by these advances has not, however, been realized, largely because of a lack of coordinated research since the GEOSECS program of the 1970s. New analytical methods that allow high-density sampling and new modeling strategies (as applied successfully during the World Ocean Circulation Experiment [WOCE] and the Joint Global Ocean Flux Study [JGOFS]) make this the right time to mount a major international research program to study the global marine biogeochemical cycles of TEIs.

The GEOTRACES mission can be expressed in two principal goals: (1) to determine global oceanic distributions of selected TEIs and (2) to evaluate the cycling of these TEIs, and thereby characterize the physical, chemical, and biological processes regulating their distributions. Research in pursuit of these goals will be organized under three themes. The first theme will examine the modern cycling of TEIs by quantifying fluxes at the principal ocean interfaces (e.g., atmosphere, continental margins, mid-ocean ridges). The second builds upon the first by determining the rates of internal cycling of TEIs within the ocean (e.g., biological uptake, chemical scavenging, physical transport). The third theme will focus on TEIs that serve as paleoceanographic proxies, to understand the factors controlling proxy distributions in the water column and sediments. A natural outcome of this work will be to build a community of marine scientists who understand the processes regulating TEIs sufficiently well to exploit them reliably in interdisciplinary studies. Another outcome will be the ability to incorporate TEI cycles into models to predict responses to future global change. cal models will be used to evaluate the relative importance of physical and biogeochemical processes and to calculate TEI fluxes from measured distributions. GEOTRACES will apply a hierarchy of

\section{GEOTRACES is motivated by the fact that TEls play critical roles in many aspects of oceanography, and by the incompleteness of our understanding of their marine biogeochemical cycles.}

GEOTRACES will be global in scope, consisting of ocean sections complemented by regional process studies. Sections and process studies will combine fieldwork, laboratory experiments, and modeling. Sections will cross regions that provide most information about sources, sinks, and internal cycling of TEIs. Although commitments have not yet been made to particular sections, priority will be given to regions of prominent TEI sources or sinks, such as dust plumes, major rivers, hydrothermal plumes, and continental margins. Sections will also sample the principal end-member water masses, as well as the major biogeographic provinces.

A fundamental principle underlying GEOTRACES is that measurement of multiple TEIs with varying behavior will provide insights into processes not attainable from study of a single TEI (see Box 1). GEOTRACES will also go beyond qualitative descriptions of sources, sinks, and internal cycling of TEIs. Numeri- model resolutions and complexity. Examples include coupled physical/biogeochemical general circulation models, box models, chemical speciation models, and inverse models. Recent advances in data assimilation and inverse modeling, for example, now allow direct data utilization methods not yet applied for determination of TEI fluxes. Inverse models promise to be an important element of ongoing and future studies of ocean circulation (e.g., the Climate Variability and Predictability program [CLIVAR]). Expanding those activities to the assimilation of TEI distributions offers a strategy to quantify TEI flux terms in marine biogeochemical cycles, including the vertical fluxes associated with TEI uptake, and regeneration by sinking particles. GEOTRACES will allow intercalibration and standardization of the analytically challenging TEI measurement methods, and provide for technology transfer and capacity building in nations that presently lack TEI measurement capability. 
The value of studying multiple TEls, and the general value of highresolution TEI data on ocean sections, is illustrated by recent results from the North Atlantic Ocean. Distributions of dissolved iron and aluminum measured at $1^{\circ}$ resolution (Figure 1 ) reveal first-order features of the sources and internal cycling of these trace elements. High surface concentrations of aluminum between the equator and $20^{\circ} \mathrm{N}$ define the location of dust deposition in the months prior to sampling. Dissolved iron concentrations are low in surface waters, but increase in the thermocline under the region with high surface aluminum concentrations. Dust deposition supplied both iron and alumi- num to surface waters, but although iron was removed from surface waters in sinking biogenic detritus, and regenerated subsequently in the thermocline, aluminum has a longer residence time and remains at the surface as a tracer of the dust deposition. Examining the two elements together tells us more about their biogeochemical cycles than could be inferred from one element alone. The sharp boundaries of the high-iron region in the thermocline also informs us that the regeneration of iron is sufficiently rapid to prevent substantial dispersion by lateral mixing.
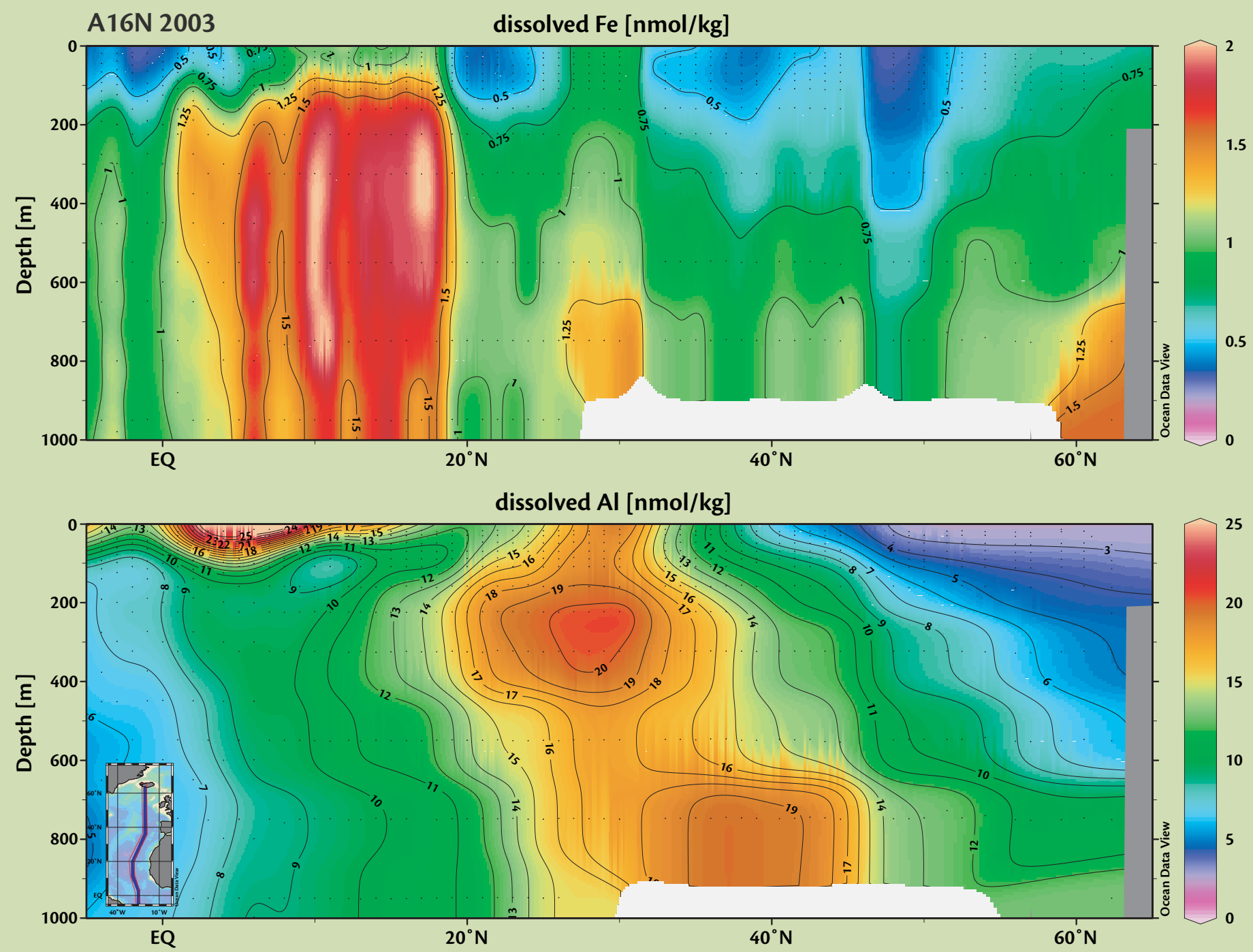

Figure 1. Distributions of dissolved iron (upper panel) and aluminum (lower panel) in the upper $1000 \mathrm{~m}$ along the CLIVAR A16 cruise track May 19 to August 11, 2003 (see inset for location). Courtesy of Chris Measures, University of Hawaii, and Bill Landing, Florida State University. 
The resources needed for this research require international cooperation. Formal planning of GEOTRACES was launched with an international workshop in April 2003 involving 85 scientists bration exercises. Working groups tasked with these responsibilities are being assembled, and meetings of these groups are being scheduled for late 2005 . These activities will be organized under the

\section{Advances in clean sampling protocols and analytical}

techniques provide unprecedented capability for measurement of a wide range of TEls. The potential afforded by these advances has not, however, been realized, largely because of a lack of coordinated research since the GEOSECS program of the 1970 s.

from 15 nations. This workshop was followed by regional/national planning workshops in Europe, North America, and the western Pacific region. In 2003, the Scientific Committee on Oceanic Research (SCOR) agreed to provide oversight for GEOTRACES, and a SCORsponsored planning group that first met in June 2004 was tasked with preparing a science plan. A draft science plan was released for community comment early in 2005, leading to revisions by the planning group. The revised Science Plan is currently under review by SCOR; a copy can be downloaded from the GEOTRACES website (see below).

On approval by SCOR of the Science Plan, the planning group will be replaced by a Science Steering Committee (SSC) to oversee implementation of the program. Early tasks for the SSC will include the creation of a data submission and management strategy, the development and distribution of standard reference materials, and the initiation of inter-cali- direction of an International Program Office, overseen by SCOR.

Preparations for fieldwork are currently in the early planning stages, with the program expected to participate in the International Polar Year (IPY) during 2007-2009. Core activities during the decade following the IPY will involve 12 to 15 ocean sections chosen to address the up process studies will focus on, for example, processes at ocean margins, the distal northern and southern limbs of meridional overturning circulation, and submarine groundwater fluxes, generally tied to the ends of ocean sections.

GEOTRACES will collaborate closely with other ocean research initiatives, including CLIVAR, the Integrated $\mathrm{Ma}$ rine Biogeochemistry and Ecosystem Research project (IMBER), the Surface Ocean-Lower Atmosphere Study (SOLAS), the Land-Ocean Interactions in the Coastal Zone project (LOICZ), the International Marine Past Global Changes Study program (IMAGES), InterRidge, MARGINS, and various modeling programs to ensure synergy among the different programs and to avoid unnecessary duplication of effort.

GEOTRACES planning activities are currently sponsored by SCOR and various national funding agencies. The members of the international planning group, the July 2005 science plan draft, and additional information is available on the GEOTRACES website: www.ldeo.

\section{GEOTRACES will collaborate closely with other ocean research initiatives...and various modeling programs to ensure synergy among the different programs and to avoid unnecessary duplication of effort.}

maximum number of processes. These activities will be run by various countries but with international representation. In addition, preparations will begin soon to initiate several process studies. Final ocean sections plus parallel and follow- columbia.edu/res/pi/geotraces/ (also accessible via www.geotraces.org). Cochairs of the planning group are Robert F. Anderson (boba@ldeo.columbia.edu) and Gideon M. Henderson (gideon. henderson@earth.ox.ac.uk). 